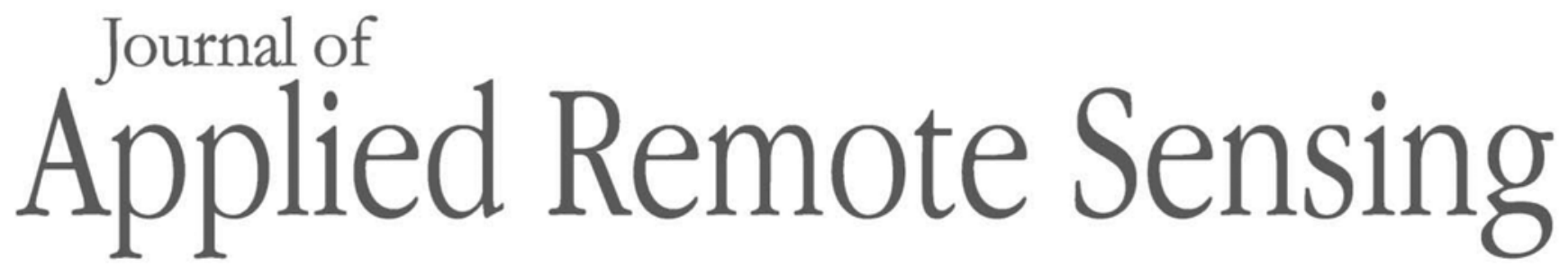

RemoteSensing.SPIEDigitalLibrary.org

\title{
AtmoCube A1: airglow measurements in the mesosphere and lower thermosphere by spatial heterodyne interferometry
}

Friedhelm Olschewski

Martin Kaufmann

Klaus Mantel

Tom Neubert

Heinz Rongen

Martin Riese

Ralf Koppmann 


\title{
AtmoCube A1: airglow measurements in the mesosphere and lower thermosphere by spatial heterodyne interferometry
}

\author{
Friedhelm Olschewski, ${ }^{\mathrm{a}, *}$ Martin Kaufmann, ${ }^{\mathrm{b}}$ Klaus Mantel, ${ }^{\mathrm{c}}$ \\ Tom Neubert, ${ }^{\mathrm{d}}$ Heinz Rongen, ${ }^{\mathrm{d}}$ Martin Riese, ${ }^{\mathrm{a}, \mathrm{b}}$ and Ralf Koppmann ${ }^{\mathrm{a}}$ \\ ${ }^{a}$ University of Wuppertal, Institute for Atmospheric and Environmental Research, \\ Wuppertal, Germany \\ ${ }^{\mathrm{b}}$ Institute of Energy and Climate Research Stratosphere (IEK-7), Research Center Juelich, \\ Juelich, Germany \\ ${ }^{c}$ Max Planck Institute for the Science of Light, Erlangen, Germany \\ ${ }^{\mathrm{d}}$ Central Institute of Engineering, Electronic Systems (ZEA-2), Research Center Juelich, \\ Juelich, Germany
}

\begin{abstract}
The Institute for Atmospheric and Environmental Research at the University of Wuppertal and the Institute of Energy and Climate Research Stratosphere at Research Center Juelich developed a CubeSat payload for atmospheric research. The payload consists of a small interferometer for the observation of airglow near $762 \mathrm{~nm}$. The line intensities of the oxygen A-band are used to derive temperatures in the mesosphere and lower thermosphere region. The temperature data will be used to analyze dynamical wave structures in the atmosphere. The interferometer technology chosen to measure the ro-vibrational structure of the $\mathrm{O}_{2}$ atmospheric band near $762 \mathrm{~nm}$ is a spatial heterodyne interferometer originally proposed by Connes in 1958. It can be designed to deliver extraordinary spectral resolution to resolve individual emission lines. The utilization of a two-dimensional imaging detector allows for recording interferograms at adjacent locations simultaneously. Integrated in a six-unit CubeSat, the instrument is designed for limb sounding of the atmosphere. The agility of a CubeSat will be used to sweep the line-of-sight through specific regions of interest to derive a three-dimensional image of an atmospheric volume using tomographic reconstruction techniques. () The Authors. Published by SPIE under a Creative Commons Attribution 4.0 Unported License. Distribution or reproduction of this work in whole or in part requires full attribution of the original publication, including its DOI. [DOI: 10 .1117/1.JRS.13.024501]
\end{abstract}

Keywords: remote sensing; $\mathrm{O}_{2}$ A-band emission; spatial heterodyne interferometer; CubeSat payload; gravity waves; tomography in the atmosphere.

Paper 180982 received Dec. 19, 2018; accepted for publication Mar. 8, 2019; published online Apr. 4, 2019.

\section{Introduction}

The University of Wuppertal and Research Center Juelich started the Development Initiative for Small Satellites Exploring Climate processes by Tomography, called DISSECT, together with German and Canadian research institutes and universities. The goal was to develop a small instrument to measure spectrally resolved radiation in the middle and upper atmospheres at high spatial resolution. The payload should be capable of deriving three-dimensional (3-D) information about atmospheric gravity wave patterns. The design should be simple with no movable parts in order to save power and to increase reliability. The instrument should fit into a small volume and should have a high optical throughput. The design should be easily adaptable to other scientific objectives.

Song et al. ${ }^{1}$ developed a new satellite observation strategy for the detection of gravity waves in the mesosphere and lower thermosphere (MLT) at high spatial resolution. This measurement technique requires an agile satellite platform to make multiangle observations of a

*Address all correspondence to Friedhelm Olschewski, E-mail: olsch@uni-wuppertal.de 
specific atmospheric volume and a spectrometer particularly suited for the detection of faint emission lines. Temperature measurements in the MLT region can be performed by the evaluation of the rotational distribution of the $\mathrm{O}_{2}$ atmospheric band near $762 \mathrm{~nm}$. Since the emitting states are sufficiently long-lived, the rotational distribution is thermalized, such that the distribution of the lines can be used to obtain kinetic temperature during day and night. ${ }^{2}$

In the past, the oxygen A-band has been used to derive global MLT temperatures using Fabry-Pérot interferometer data from the Upper Atmospheric Research Satellite-HighResolution Doppler Imager ${ }^{3}$ and grating spectrometer data from Odin Optical Spectrograph and InfraRed Imaging System. ${ }^{4}$

Since the temperature measurement presented in this paper relies on relative intensities only, the requirements for monitoring the radiometric performance of such an instrument are much more relaxed than for measurement strategies that depend on absolute intensities. Another advantage is that the $\mathrm{O}_{2}$ A-band emits at wavelengths below $1 \mu \mathrm{m}$, so silicon-based detectors operating at ambient or moderately cooled conditions can be used as image sensors. This reduces power consumption, mass, and costs of such an instrument significantly. ${ }^{5}$

For the study of faint radiation from the atmosphere, an interferometer has a significant advantage over conventional grating spectrometers. The throughput is typically more than 2 orders of magnitude larger than for grating spectrometers of the same size. Since a spatial heterodyne interferometer (SHI) has no moving parts, it can be built as a monolithic block, which makes it very attractive for space instruments. Combined with a two-dimensional imaging detector, it records a set of interferograms from a scene in the atmosphere with one dimension providing the spectral information while the spatial information is found in the second dimension.

\section{Importance of the Mesopause Region for Climate Studies}

The mesopause region is where space weather meets Earth's atmosphere and vice versa.

Studying processes in the upper atmosphere will help to improve the understanding of potential anthropogenic effects on the climate system, which is more and more perceived by researchers. ${ }^{6,7}$ The most uncertain aspect of climate modeling lies in the representation of unresolved small-scale processes. ${ }^{8}$ Gravity wave drag and its sensitive interaction with large-scale dynamics can no longer be neglected.

Especially, the MLT region is dominated by waves that influence the wind and temperature fields of this altitude range. Coupling processes initiated by gravity waves in the middle atmosphere have increasing importance for the modeling of the climate system and represent one of the larger uncertainties in this field, because gravity waves are one of the least explored dynamical processes in the middle and upper atmospheres. They are mostly generated in the troposphere, propagate vertically and horizontally, carrying momentum from the troposphere up to the stratosphere, mesosphere, and even to the thermosphere. The wavelength range of these waves is in the order of several kilometers in the vertical and from tens to hundreds of kilometers in the horizontal. When gravity waves break, they deposit their momentum at that particular region of the atmosphere. This phenomenon is the key driver for the summer-to-winter pole circulation at the mesopause and one of the most uncertain parameters in climate models.

The National Center for Atmospheric Research Whole Atmosphere Community Climate Model was used to investigate the dynamical influence of the lower and middle atmospheres on the upper MLT. ${ }^{9}$ For the coupling, a strong dependency on the method used for representing gravity wave drag was found. This demonstrates the role of gravity waves in transporting the variability of the troposphere into the MLT. The diversity of gravity wave generation and breaking in the atmosphere is described by Kim et al. ${ }^{10}$

As shown in Fig. 1, the diversity and multifaceted structure of gravity waves in the mesopause region imprinted on noctilucent clouds at about $83 \mathrm{~km}$ can be observed from ground. ${ }^{11}$ The anthropogenic impact on the long-term evolution of noctilucent clouds has been investigated by different researchers. ${ }^{12,13}$ Global observations of spatial, temporal, and spectral distributions of gravity waves are still one of the biggest challenges in climate research. 


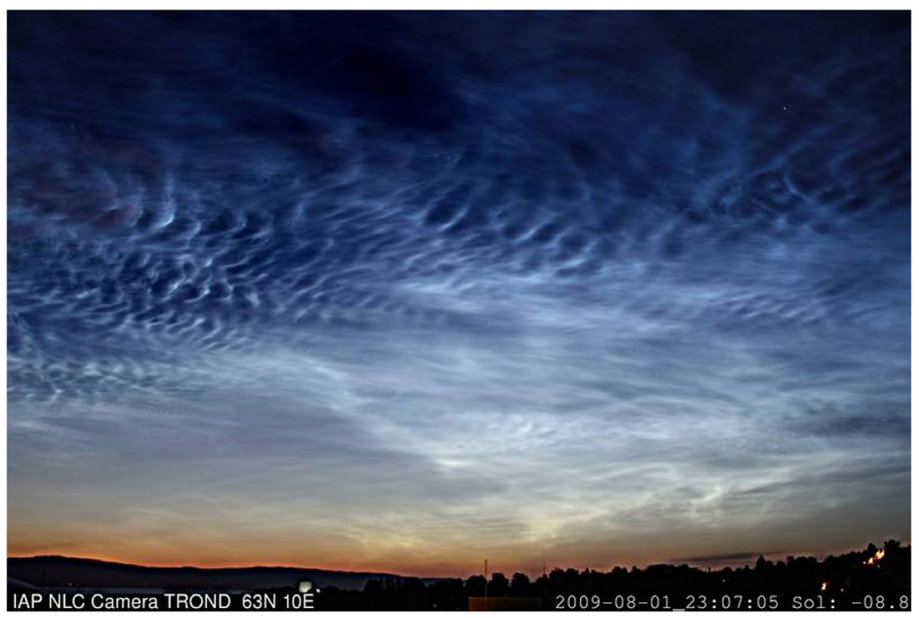

Fig. 1 Gravity waves imprinted on noctilucent clouds as observed by the Institute of Atmospheric Physics noctilucent cloud camera in 2009.

\section{Temperature Derivation from Oxygen A-Band Emissions}

Gravity waves can be detected by measuring the temperature field in the atmosphere. ${ }^{14} \mathrm{~A}$ simulation of a typical temperature distribution generated by a gravity wave is shown in Fig. 2 for the altitude range between 86 and $104 \mathrm{~km}$. The black lines correspond to the initial temperature field assumed for the retrieval. The color code refers to the reconstruction of the predetermined temperature field, when a specific measurement mode is applied. The horizontal coverage shown in the figure is several hundred kilometers. Several wave cycles can be seen with a wave amplitude of the temperature variation of up to $\pm 5 \mathrm{~K}$.

Based on the observed temperature field, the wavelength, the amplitude, and the phase of the wave can be determined. One option to derive the temperature distribution in the upper atmosphere is to measure the oxygen A-band emission near $762 \mathrm{~nm}$. The long radiative lifetime ensures that the molecules are in rotational equilibrium with the ambient atmosphere, such that rotational and ambient temperatures are identical. ${ }^{15}$

The vertical distribution of A-band emissions is given by Kaufmann et al. ${ }^{5}$ Figure 3(a) gives the spectral distribution of the emission lines with selected lines marked in different colors. The line intensities are calculated based on equations given by Song et al. ${ }^{14}$ Since the rotational structure of the $\mathrm{O}_{2}$ A-band follows the Boltzmann law, the ratio of different rotational lines allows for temperature retrieval without the need for a precise absolute radiometric calibration of the instrument. The temperature dependence of different lines is shown in Fig. 3(b).

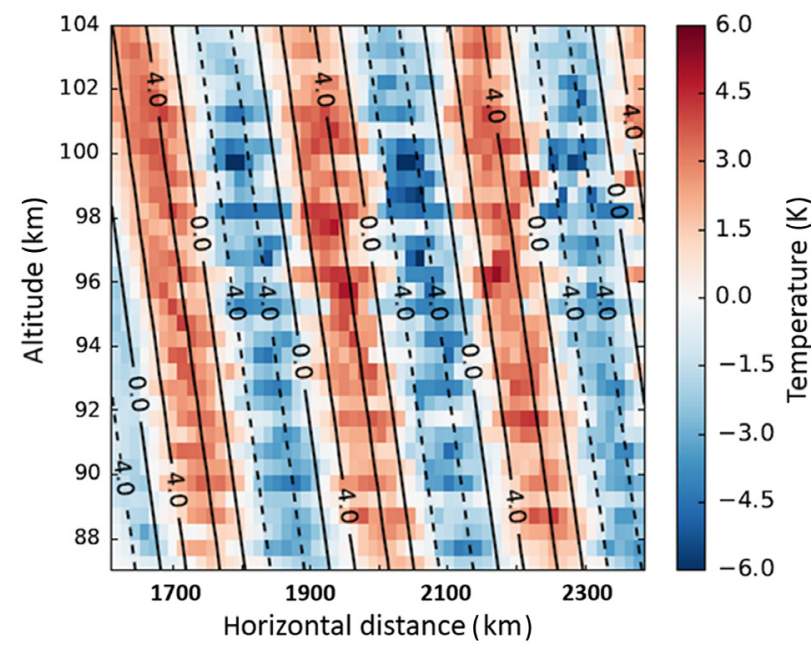

Fig. 2 Simulation of a typical temperature distribution generated by a gravity wave between 86 and $104 \mathrm{~km}$. 

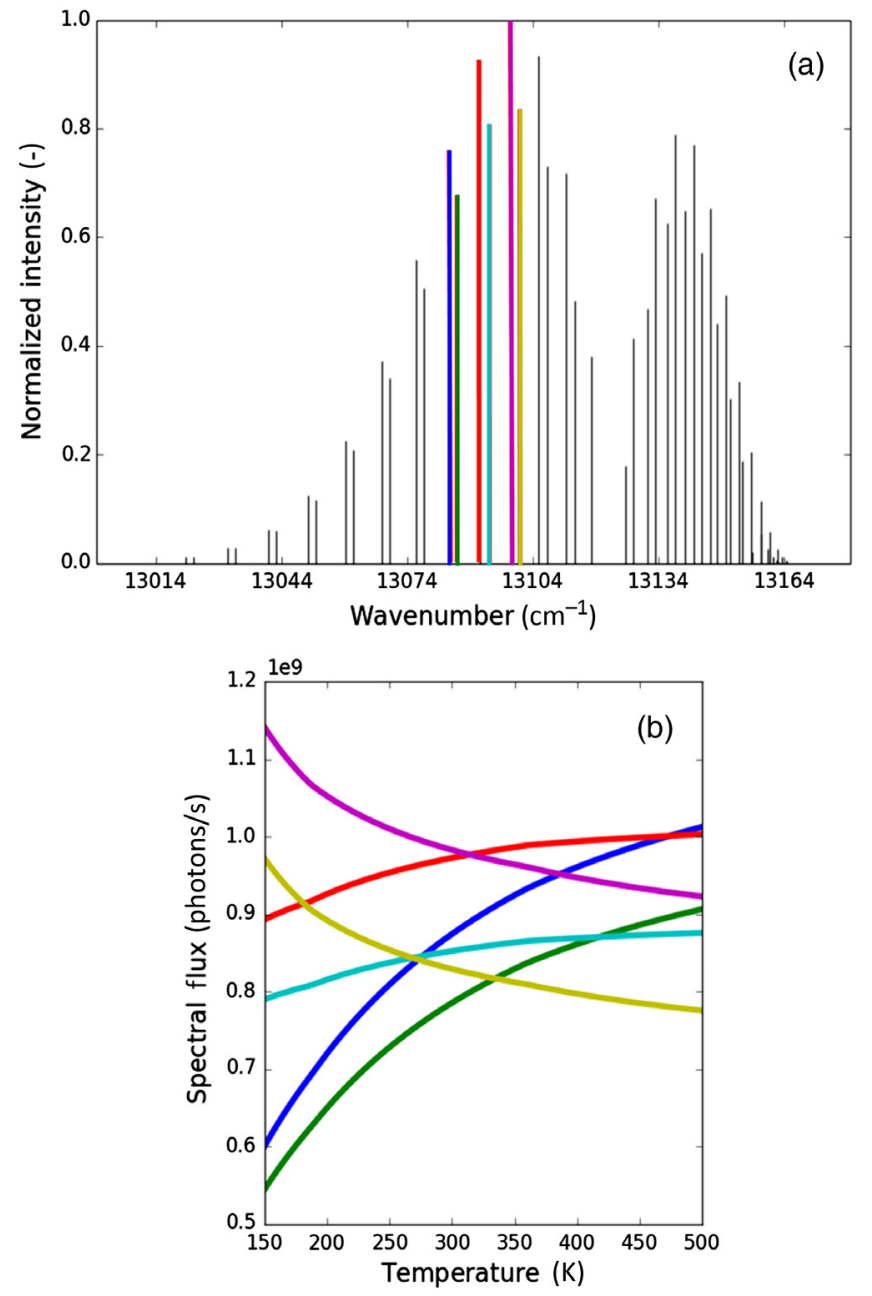

Fig. $3 \mathrm{O}_{2}$ A-band: (a) rotational structure and (b) temperature dependence of individual lines.

\section{Spatial Heterodyne Interferometer for Remote Sensing}

The spectrometer technology chosen to measure the ro-vibrational structure of the $\mathrm{O}_{2}$ atmospheric band is an SHI, originally proposed by Connes ${ }^{16}$ named SISAM. Recent developments in imaging technology and optical materials allow for useful applications of spatial heterodyne interferometry. An SHI is similar to a Michelson interferometer, where the mirrors are replaced by tilted diffraction gratings (see Fig. 4). Due to the dispersion of the gratings, diffracted light waves will show a wavefront tilt depending on the wavelength. This tilt leads to interference fringes in the detector plane with a characteristic spatial frequency for each incoming wavelength. The resulting interference pattern is the incoherent superposition of these patterns and can be analyzed by applying a numerical Fourier transform. Thus, the original wavelength spectrum can be determined.

An SHI can be designed to deliver excellent spectral resolution to resolve individual emission or absorption lines while the optical throughput is still high.

The tilt angle of the gratings with respect to the incoming wave front is called Littrow angle $\theta_{\mathrm{L}}$, which is the key design parameter of an SHI. Light at the Littrow wavenumber $\sigma_{\mathrm{L}}$ is returned in the same direction as the incoming path, as described by the following grating equation for diffraction order 1 :

$$
\sigma_{\mathrm{L}}=\frac{1}{2 d \sin \theta_{\mathrm{L}}},
$$

where $1 / d$ is the grating groove density. 


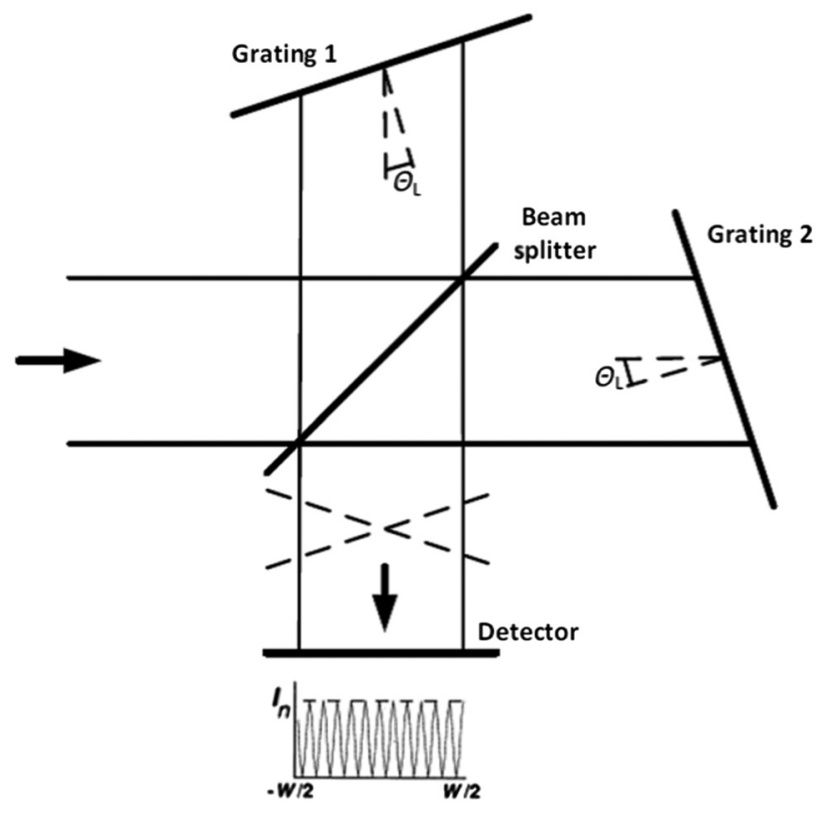

Fig. 4 Schematic of an SHI.

Waves with wavenumbers deviating from the Littrow wavenumber are diffracted back from the gratings at a slightly different angle, introducing the wavelength-dependent tilt that encodes the spectral information. The spatial frequency $\nu_{F}$ seen at the detector depends on the difference between the emission wavenumber $\sigma$ and the Littrow wavenumber $\sigma_{\mathrm{L}}$. The following equation expresses this relationship:

$$
v_{F}=4\left[\frac{\left(\frac{m}{2 d \sigma_{\mathrm{L}}}\right)}{\sqrt{1-\left(\frac{m}{2 d \sigma_{\mathrm{L}}}\right)^{2}}}\right]\left(\sigma-\sigma_{\mathrm{L}}\right),
$$

where $m$ is the diffraction order and $1 / d$ is the grating groove density.

The acceptance angle of an SHI can be increased significantly, if prisms are inserted into the two interferometer arms. This field-widening configuration was first implemented for upper atmospheric temperature measurements by Hilliard and Shepherd ${ }^{17}$ using a Michelson interferometer. Depending on the actual design, the prisms increase the throughput of an SHI by 1 to 2 orders of magnitude.

As an SHI has no moving parts, it can be built as a monolithic block, which makes it very attractive for atmospheric measurements, especially from space. Figure 5 illustrates the AtmoCube A1 SHI design (a) and provides an image of the prototype (b). With dimensions of $38 \mathrm{~mm} \times 38 \mathrm{~mm} \times 27 \mathrm{~mm}$, it fits well into a CubeSat. ${ }^{18}$

According to Lenzner and Diels, ${ }^{19}$ the resolving power $R$ of an SHI is defined as

$$
R=\frac{2 W \tan \theta_{\mathrm{L}}}{d}
$$

where $W$ is the width of the illuminated area on the gratings and $1 / d$ is the grating groove density.

A grating groove density of $1 / d=1200$ lines per $\mathrm{mm}$ and an illuminated width on the gratings of $W=15 \mathrm{~mm}$ lead to a maximum resolving power of about 18,500.

To determine the expected signal-to-noise ratio of the instrument for a given integration time, the amount of incoming light, which is available in the modulated part of the interferogram, and the noise of the detector have been calculated. In an SHI, 50\% of the incoming radiation is lost at 


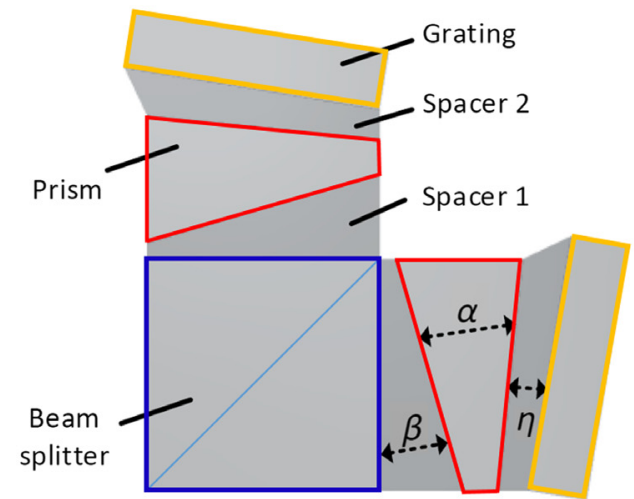

(a)

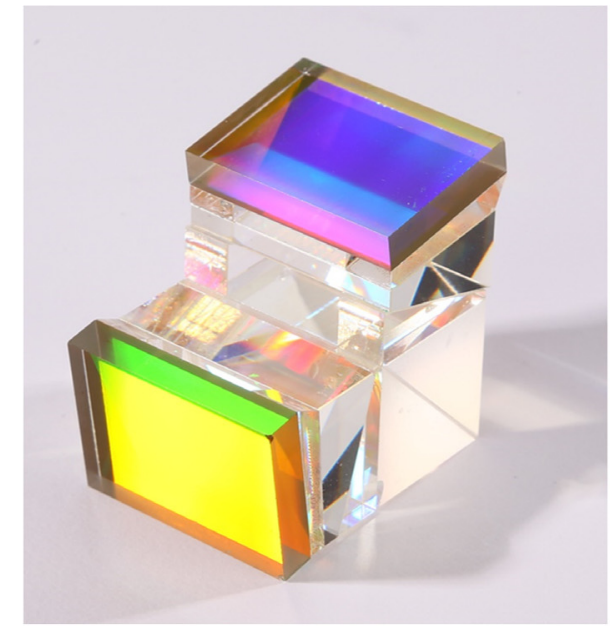

(b)

Fig. 5 AtmoCube A1 SHI prototype: (a) design and (b) prototype.

the beam splitter. Due to the fact that typical gratings have an efficiency of about $70 \%$ at $765 \mathrm{~nm}$, another $30 \%$ of the radiation is not available. Misalignments and aberrations of optical components reduce the contrast of the interferogram furthermore so that only about $10 \%$ to $30 \%$ of the atmospheric signal can be expected in the modulated part of the interferogram. The detector image of simulated interferograms from a single emission line in a focused configuration is shown in Fig. 6. The simulated image size is $400 \times 400$ pixels with a pixel size of $10 \mu \mathrm{m} \times 10 \mu \mathrm{m}$.

\section{AtmoCube A1 Limb Sounder}

\subsection{Optical Design}

To measure vertically resolved interferograms, a front optics is necessary for imaging the atmospheric scene onto the gratings. A large aperture with a diameter of $70 \mathrm{~mm}$ leads to a high etendue (throughput) of $0.02 \mathrm{~cm}^{2} \mathrm{sr}$, and a bandpass filter selects a narrow wavelength range $(3.4 \mathrm{~nm})$ in order to avoid adverse photons in the optical system. The optical design of the AtmoCube A1 prototype is shown in Fig. 7. The detector optics images the illuminated area of the gratings onto the limb sounding detector (LSD), creating a set of interferograms. An additional stray light baffle in front of the system shall protect the instrument from inadvertent radiation reaching the detector.

Depending on the launch opportunities, a wide range of orbit altitudes are acceptable. The front optics has a field of view of $1.3 \mathrm{deg}$, which corresponds to a vertical range of 50 to $80 \mathrm{~km}$ at 


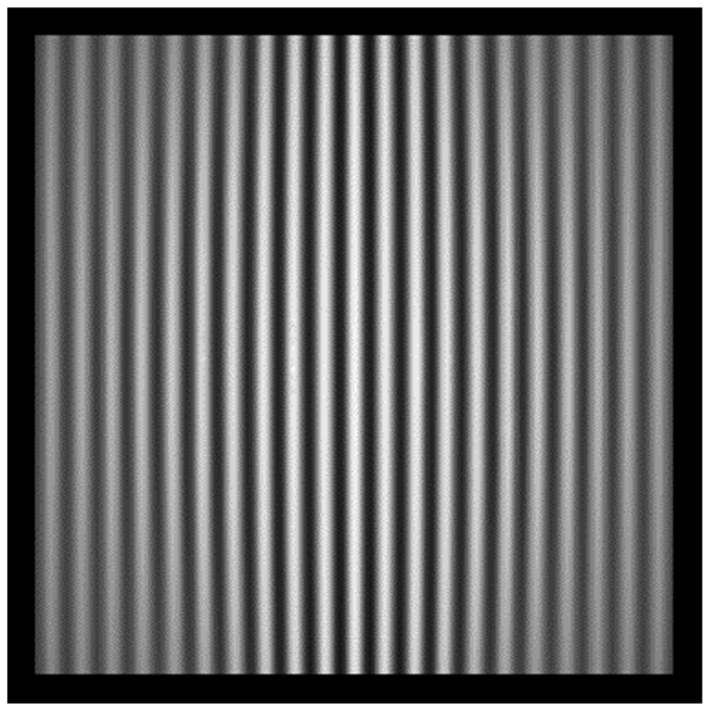

Fig. 6 Detector image $(400 \times 400$ pixel $)$ of simulated interferograms.

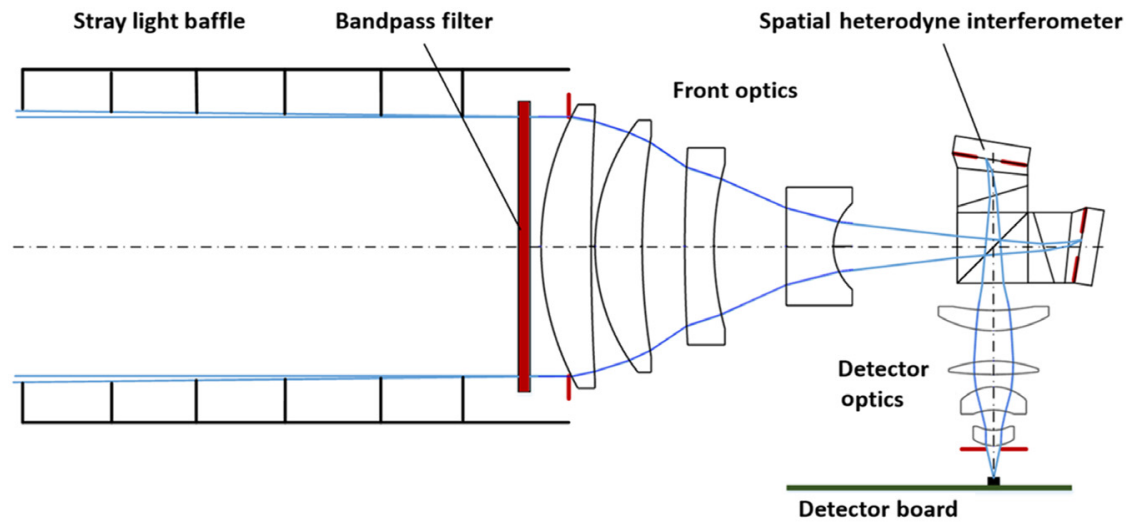

Fig. 7 AtmoCube A1 prototype optical design.

the Earth limb with orbit altitudes between 400 and $1000 \mathrm{~km}$. Assuming an orbit altitude of $600 \mathrm{~km}$, the front optics is designed in such a way that an area of $60 \mathrm{~km} \times 60 \mathrm{~km}$ at the limb in the range of 60 to $120 \mathrm{~km}$ can be observed (see Fig. 8). Spatial binning is variable and depends on emission intensity and integration time. A single detector pixel sees an atmospheric element of $\sim 200 \mathrm{~m}$ in the vertical. The envisaged attitude resolution is $1.5 \mathrm{~km}$. Since the distance between the satellite and the tangent point is between 2300 and $3500 \mathrm{~km}$, a highly precise attitude control in the subarcmin range is required.

\subsection{Thermally Controlled Image Sensor}

Since the airglow is a faint phenomenon, high-quality imaging in extremely low light conditions is mandatory. The back-side illuminated scientific CMOS image sensor GSENSE 400 BSI with 4.2 megapixel resolution is used. The large optical area $(22.5 \mathrm{~mm} \times 22.5 \mathrm{~mm})$ matches well with the image size of the atmospheric scene on the gratings. The sensor has an extremely low readout noise of $1.6 \mathrm{e}$ - and a high quantum efficiency of about $70 \%$ at $760 \mathrm{~nm}$. The integration time will be up to several seconds depending on the atmospheric conditions (daytime or nighttime). 


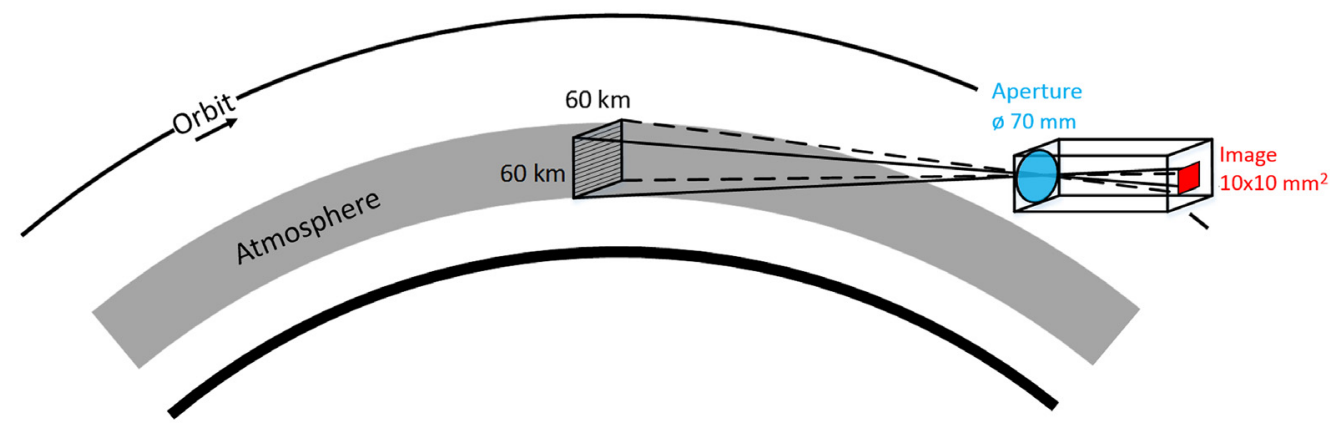

Fig. 8 Limb view of the AtmoCube A1 satellite.

The detector shall be operated at a temperature between $-30^{\circ} \mathrm{C}$ and $0^{\circ} \mathrm{C}$ in order to increase the signal-to-noise ratio. To maintain the temperature in this range during operation, a thermal control unit (TCU) is needed. A passive radiator facing deep space will be installed on one side of AtmoCube A1.

\subsection{AtmoCube A1 Payload Electronics}

The AtmoCube A1 payload electronics controls two imagers, the LSD and the albedo cloud detector (ACD). It consists of two functional blocks, the proximity electronics (PXE) for directly interfacing to several detector ICs and the front-end electronics (FEE) with an embedded controller, which serves as the central processing system. The FEE handles signals from the CubeSat bus to start the measurement, to acquire detector data, to provide data preprocessing (e.g., data binning), and to transfer data to the command and data handling (C\&DH) subsystem. Figure 9 shows the block diagram of the AtmoCube A1 payload electronics.

The design uses state-of-the-art, commercial-off-the-shelf components with an adapted approach to achieve radiation-tolerant characteristics considering smart requirements and a subset of mitigation techniques. The electronics combines hardware and software redundancies to improve system availability and reliability for long-life missions. ${ }^{20}$

The system controller of the FEE is based on a system-on-chip (SoC) architecture, which combines reconfigurable logic with a dual-core processor system. Two scientific CMOS detectors are applied. The GSENSE 400 BSI from GPixel Inc. is used as LSD and the HWK1910A from BAE Systems as ACD, which operate independently and are handled in parallel by the system controller.

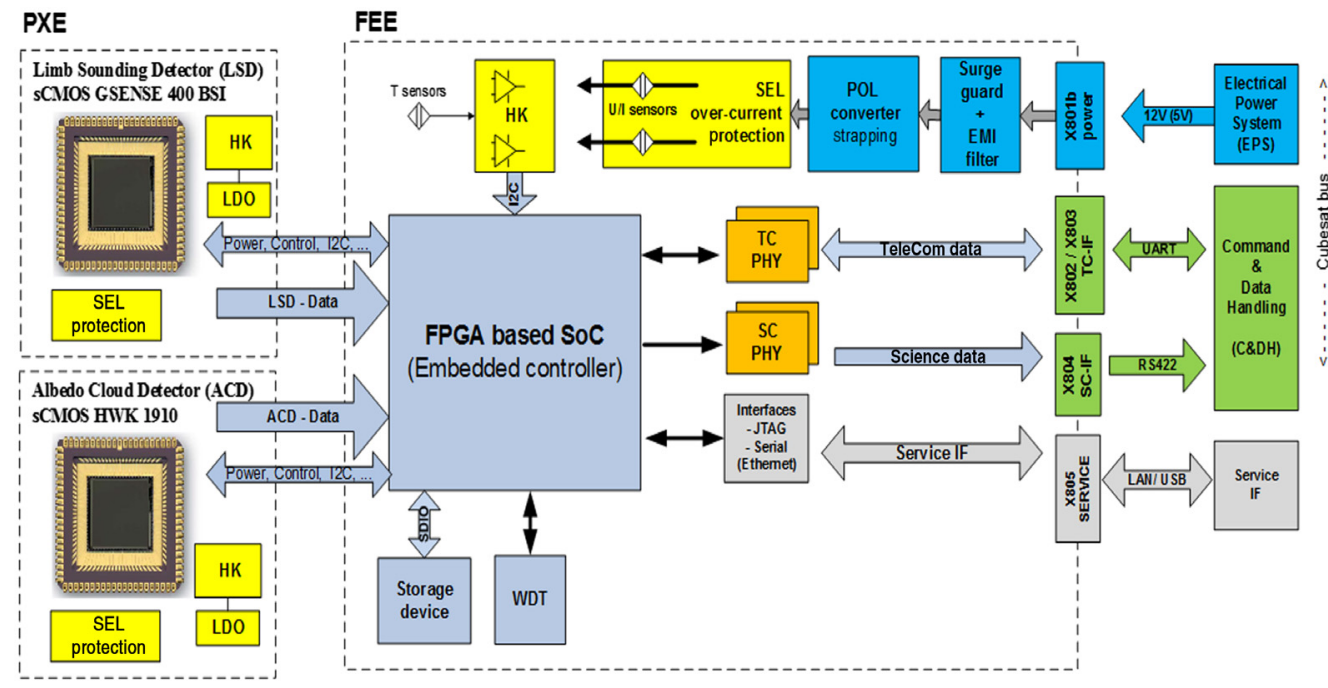

Fig. 9 Block diagram of the AtmoCube A1 payload electronics (for details, see Neubert et al ${ }^{20}$ ). 
The controller boots from an on-module flash memory or a solid-state disk device, which provides a reference boot image in redundancy. The operation of the system controller is checked by a watchdog timer (WDT), which triggers a reset signal in case of a firmware violation or logic malfunction. Processor memory failures are mitigated using memory chips with integrated error code correction, periodic memory pattern, and cycling redundancy checks on selected data structures. The logic configuration is placed in triple-module-redundancy, and the majority voters generate an index signal in case of a discrepancy.

At power-up, a build-in-self-test (BIST) sequence checks all power rails, the attached interfaces, and subsystems. The result of the BIST and single event upset detection procedures are reported in a status register that is included in the housekeeping data package. Furthermore, the image sensors, PXE, and FEE are protected against single event latch-up (SEL) using overcurrent protection circuits. The payload electronics can operate from common CubeSat bus voltages and generate the internal supplies with redundant point-of-load (POL) converters.

The CubeSat space protocol will be used to achieve a standardized communication via CAN, I2C, or RS-422 between common CubeSat subsystem modules. In principle, the communication is divided into two channels: (1) a telecommand interface to control the instrument and acquire housekeeping values and (2) a science data interface to send the detector data to the C\&DH. Each channel is implemented as a redundant logic core and can be a combination of different physical interfaces in case of limited resources.

Additional interfaces are implemented for ground operations, testing, and qualification: an Ethernet interface as image streaming for real-time calibration procedures, a USB interface as service terminal, and an in-system programming interface for firmware updates. These components are independently powered by the ground support equipment and in "cold standby" during the AtmoCube A1 mission.

\section{AtmoCube A1 Design}

AtmoCube A1 limb sounder consists of the optics, the electronics, and the TCU as described in Sec. 5. The mass of the payload is $<3 \mathrm{~kg}$ with a power consumption of $<8 \mathrm{~W}$. The data rate can be reduced to $300 \mathrm{kbit} / \mathrm{s}$ by pixel binning. Since limb viewing measurements require highly precise attitude control and determination, attitude control accuracy in pitch shall be about $0.003 \mathrm{deg}$, which corresponds to an uncertainty in altitude determination of $140 \mathrm{~m}$ from a $600-\mathrm{km}$ orbit. The detailed payload specifications are given in Table 1.

The AtmoCube A1 payload is designed for a six-unit (6U) CubeSat. In addition to the main payload, an albedo cloud camera (ACC) for the detection of stray light reflected from tropospheric clouds is included in the AtmoCube concept.

As in all CubeSat systems, solar panels, an electrical power system, an attitude determination and control system (ADCS), an onboard computer, and an S-band antenna for communication are needed. As shown in Fig. 10, all these components are compiled to fit into a $6 \mathrm{U}$ CubeSat.

\section{AtmoCube A1 Operational Modes}

The agility of a CubeSat shall be used to focus the measurements on specific regions from different viewing angles. To measure gravity waves at high spatial resolution making multiangle observations, CubeSat agility can be used by sweeping the line-of-sight through specific regions of interest to derive a 3-D image of an atmospheric volume using tomographic reconstruction techniques. Typical slew rate for a $6 \mathrm{U}$ CubeSat of $5 \mathrm{deg} / \mathrm{s}$ to $10 \mathrm{deg} / \mathrm{s}$ can be achieved by the ADCS. Two potential observation modes for a single spacecraft mission are shown in Fig. 11. For illustration purposes, an image of the Moderate Resolution Imaging Spectroradiometer on NASA's Terra satellite is used as background, where a large-scale, overlapping wave pattern in the sun glint region of the Arabian Sea was captured. The red inverted triangles mark the tangent points of the limb measurements. An integration time of $10 \mathrm{~s}$ and a slew rate of $5 \mathrm{deg} / \mathrm{s}$ correspond to a sampling rate of $100 \mathrm{~km}$ at the tangent point. 
Olschewski et al.: AtmoCube A1: airglow measurements in the mesosphere and lower thermosphere...

Table 1 AtmoCube A1 limb sounder specifications.

\begin{tabular}{|c|c|}
\hline \multicolumn{2}{|c|}{ Optics } \\
\hline Aperture & $\varnothing 70 \mathrm{~mm}$ \\
\hline Etendue (throughput) & $0.02 \mathrm{~cm}^{2} \mathrm{sr}$ \\
\hline Atmospheric limb image & $60 \mathrm{~km} \times 60 \mathrm{~km}$ \\
\hline Altitude range & 60 to $120 \mathrm{~km}$ \\
\hline Vertical resolution & $1.5 \mathrm{~km}$ \\
\hline Spectral range & $3.4 \mathrm{~nm}(761.9$ to $765.3 \mathrm{~nm})$ \\
\hline Maximum resolving power $(\lambda / \Delta \lambda)$ & 18,500 \\
\hline \multicolumn{2}{|c|}{ Detector } \\
\hline CMOS image sensor & GSENSE $400 \mathrm{BSI}$ \\
\hline Number of pixels & $2048(H) \times 2048(V)$ \\
\hline Quantum efficiency & 0.7 at $760 \mathrm{~nm}$ \\
\hline Thermal control & Deep space radiator \\
\hline \multicolumn{2}{|c|}{ Instrument } \\
\hline Mass & $<3 \mathrm{~kg}$ \\
\hline Power & $<8 \mathrm{~W}$ \\
\hline Data rate after binning & $300 \mathrm{kbit} / \mathrm{s}$ \\
\hline Attitude control accuracy & 0.003 deg (pitch) \\
\hline Operating temperature & $-40^{\circ} \mathrm{C}$ to $50^{\circ} \mathrm{C}$ \\
\hline
\end{tabular}

The final goal is a constellation of several satellites that view a region of interest from different perspectives. ${ }^{21}$ An artist's concept of an AtmoCube constellation is given in Fig. 12. The details on the optimal satellite constellation will follow at a later stage. Final constellation also depends on the number of satellites, their maneuverability, and other factors.

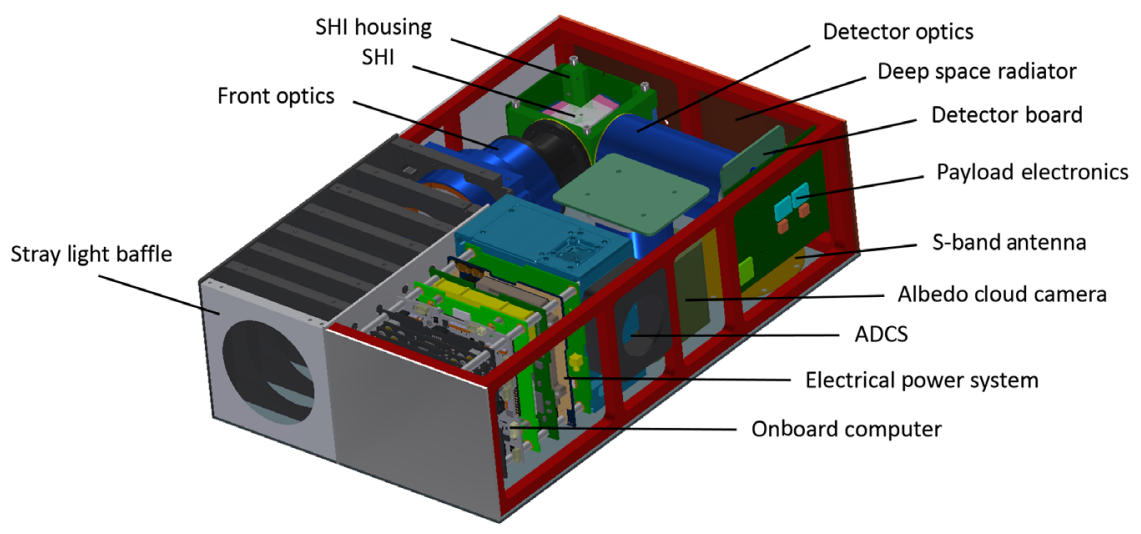

Fig. 10 AtmoCube A1 satellite design (6U CubeSat). 

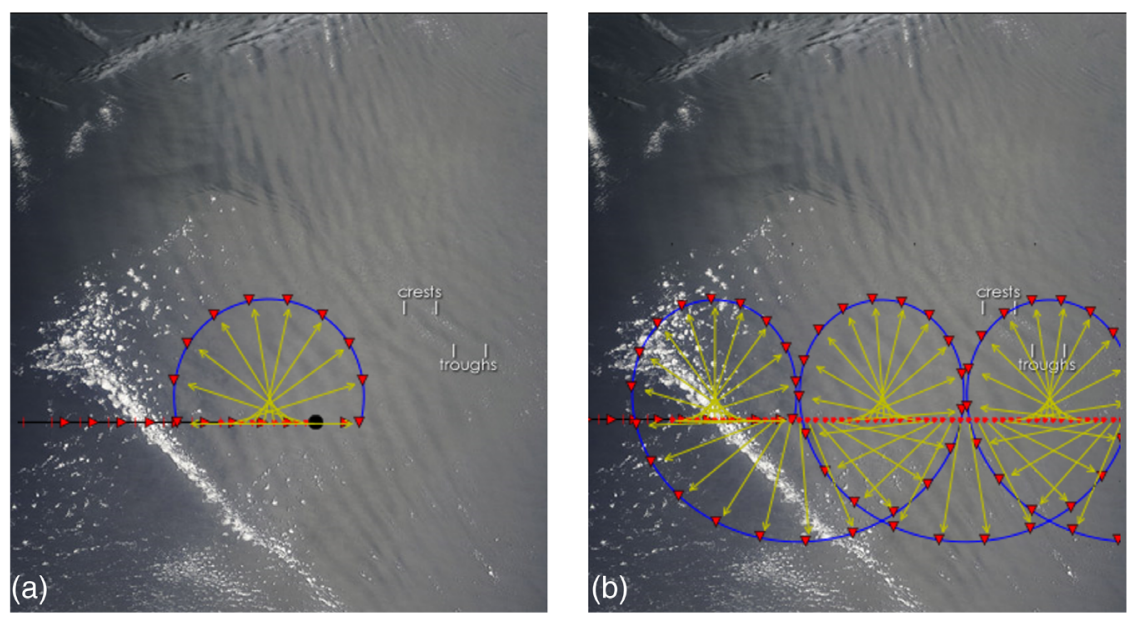

Fig. 11 AtmoCube A1 operational modes: (a) slew mode and (b) spin mode.

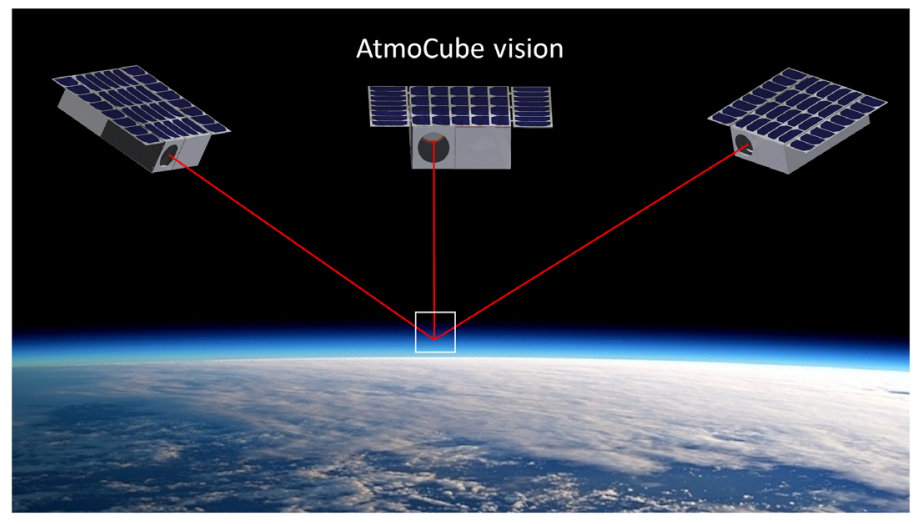

Fig. 12 Artist's concept of an AtmoCube constellation.

\section{Conclusion}

As the features of the MLT region are valuable proxies of climate change, advanced measurement techniques are needed to provide detailed information about the dynamical disposition of the upper atmosphere. To measure 3-D gravity wave fields at those altitudes is still a challenge for satellite instruments. An SHI has been developed to measure oxygen A-band emissions between 60 and $120 \mathrm{~km}$ altitude globally. As an SHI has no moving parts, it can be built as a monolithic block, which makes it very attractive for atmospheric measurements, especially from space. It fits into a small volume and still has an excellent throughput concurrently with high spectral resolution. Interferograms from different altitudes can be measured simultaneously. The agility of a CubeSat shall be used to detect dynamical structures in the MLT region by a tomographic approach. The final goal is a constellation of several satellites that view a region of interest from different perspectives.

\section{References}

1. R. Song et al., "Tomographic reconstruction of atmospheric gravity wave parameters from airglow observations," Atmos. Meas. Tech. 10, 4601-4612 (2017).

2. D. E. Burch and D. A. Gryvnak, "Strengths, widths, and shapes of the oxygen lines near 13, $100 \mathrm{~cm}^{-1}$ (7620 Å)," Appl. Opt. 8, 1493-1499 (1969).

3. D. A. Ortland et al., "Remote sensing of mesospheric temperature and $\mathrm{O}_{2}\left({ }^{1} \Sigma\right)$ band volume emission rates with the high-resolution Doppler imager," J. Geophys. Res. 103(D2), 1821-1835 (1998). 
4. P. Sheese et al., "Temperatures in the upper mesosphere and lower thermosphere from OSIRIS observations of $\mathrm{O}_{2}$ A-band emission spectra," Can. J. Phys. 88, 919-925 (2010).

5. M. Kaufmann et al., "A highly miniaturized satellite payload based on a spatial heterodyne spectrometer for atmospheric temperature measurements in the mesosphere and lower thermosphere," Atmos. Meas. Tech. 11, 3861-3870 (2018).

6. G. Beig, "Long-term trends in the temperature of the mesosphere/lower thermosphere region: 1. Anthropogenic influences," J. Geophys. Res. Atmos. 116, A00H11 (2011).

7. R. A. Vincent, "The dynamics of the mesosphere and lower thermosphere: a brief review," Prog. Earth Planet. Sci. 2, 4 (2015).

8. T. G. Shepherd, "Atmospheric circulation as a source of uncertainty in climate change projections," Nat. Geosci. 7, 703-708 (2014).

9. A. K. Smith et al., "On the dynamical control of the mesosphere-lower thermosphere by the lower and middle atmosphere," J. Atmos. Sci. 74, 933-947 (2017).

10. Y.-J. Kim, S. D. Eckermann, and H.-Y. Chun, "An overview of the past, present and future of gravity-wave drag parametrization for numerical climate and weather prediction models," Atmos. Ocean 41, 65-98 (2003).

11. G. Baumgarten and D. C. Fritts, "Quantifying Kelvin-Helmholtz instability dynamics observed in noctilucent clouds: 1. Methods and observations," J. Geophys. Res. Atmos. 119, 9324-9337 (2014).

12. G. E. Thomas et al., "Relation between increasing methane and the presence of ice clouds at the mesopause," Nature 338, 490-492 (1989).

13. F.-J. Lübken, U. Berger, and G. Baumgarten, "On the anthropogenic impact on long-term evolution of noctilucent clouds," Geophys. Res. Lett. 45, 6681-6689 (2018).

14. R. Song et al., "Three-dimensional tomographic reconstruction of atmospheric gravity waves in the mesosphere and lower thermosphere (MLT)," Atmos. Meas. Tech. 11, 3161-3175 (2018).

15. D. A. Long et al., " $\mathrm{O}_{2}$ A-band line parameters to support atmospheric remote sensing," J. Quant. Spectrosc. Radiat. Transfer 111, 2021-2036 (2010).

16. P. Connes, "Spectromètre interférential à selection par l'amplitude de modulation," J. Phys. Radium 19, 215-222 (1958).

17. R. L. Hilliard and G. Shepherd, "Wide-angle Michelson interferometer for measuring Doppler line widths," J. Opt. Soc. Am. 56, 362-369 (1966).

18. M. Deiml, "Development of a small satellite remote sensing payload for passive limb sounding of the atmospheric oxygen emission," PhD Thesis, University of Wuppertal (2017).

19. M. Lenzner and J.-C. Diels, "Concerning the spatial heterodyne spectrometer," Opt. Express 24, 1829-1839 (2016).

20. T. Neubert et al., "System-on-module based long-life electronics for remote sensing imaging with CubeSat in low-earth-orbits," J. Appl. Remote Sens. (2019).

21. F. Olschewski et al., "A novel CubeSat payload for airglow measurements in the mesosphere and lower thermosphere," Proc. SPIE 10769, 1076903 (2018).

Biographies of the authors are not available. 